A. Egges, S. Kshirsagar, N. M. Thalmann. A Model for Personality and Emotion Simulation. Knowledge-Based Intelligent Information \& Engineering Systems (KES2003). 2003. 


\title{
A Model for Personality and Emotion Simulation
}

\author{
Arjan Egges, Sumedha Kshirsagar, and Nadia Magnenat-Thalmann \\ MIRALab - University of Geneva \\ 24, Rue General-Dufour,1211 Geneva, Switzerland \\ \{egges, sumedha, thalmann\}@miralab.unige.ch \\ WWW home page: http://www.miralab.ch
}

\begin{abstract}
This paper describes a generic model for personality, mood and emotion simulation for conversational virtual humans. We present a generic model for describing and updating the parameters related to emotional behaviour. Also, this paper explores how existing theories for appraisal can be integrated into the framework. Finally we describe a prototype system that uses the described models in combination with a dialogue system and a talking head with synchronised speech and facial expressions.
\end{abstract}

\section{Introduction}

With the emergence of $3 \mathrm{D}$ graphics, we are now able to create very believable $3 \mathrm{D}$ characters that can move and talk. However, an important part often missing in this picture is the definition of the force that drives these characters: the individuality. In this paper we explore the structure of this entity as well as its link with perception, dialogue and expression. In emotion simulation research so far, appraisal is popularly done by a system based on the OCC model [1]. This model specifies how events, agents and objects from the universe are appraised according to an individual's goals, standards and attitudes. These three (partly domain-dependent) parameters determine the 'personality' of the individual. More recent research indicates that personality can be modelled in a more abstract, domain-independent way $[2,3]$. In this paper we will investigate the relationship between such personality models and the OCC model.

The effect of personality and emotion on agent behaviour has been researched quite a lot, whether it concerns a general influence on behaviour [4], or a more traditional planning-based method [5]. Various rule based models [6] and probabilistic models [7] have been reported in the past. How behaviour should be influenced by personality and emotion depends on the system that is used and it is out of the scope of this paper.

The effect of personality and emotion will also have an effect on expression (speech intonation, facial expressions, etc.) is partly deatl with in Kshirsagar et al. in [8], which describes a system that simulates personalized facial animation with speech and expressions, modulated through mood. There have been very few researchers who have tried to simulate mood $[9,10]$. 
Figure 1 depicts how we view the role of personality and emotion as a glue between perception, dialogue and expression. Perceptive data is interpreted on an emotional level by an appraisal model. This results in an emotion influence that determines, together with the personality what the new emotional state and mood will be. An intelligent agent uses the emotional state, mood and the personality to create behaviour.

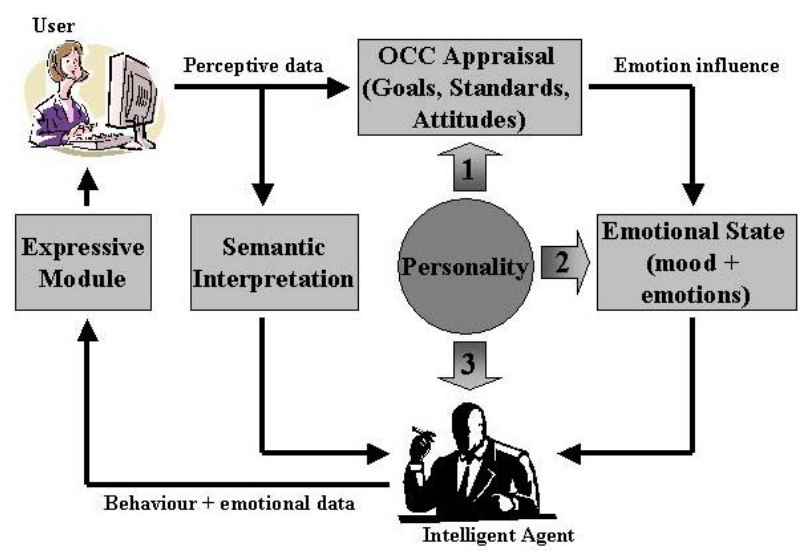

Fig. 1. Overview of the emotional state and personality in an intelligent agent framework.

This paper is organized as follows. Section 2 and Section 3 present the generic personality, mood and emotion model. In Section 4, we investigate the relationship between the OCC model and personality. Finally, we present an example of how to use our model with an expressive virtual character ${ }^{1}$.

\section{A Generic Personality and Emotion Model}

The intention of this section is to introduce the concepts that we will use in our model. In the next section we will explain how these concepts interact. We will first start by defining a small scenario.

Julie is standing outside. She has to carry a heavy box upstairs. A passing man offers to help her carry the box upstairs. Julies personality has a big influence on her perception and on her behaviour. If she has an extravert personality, she will be happy that someone offers her some help. If she has a highly introvert and/or neurotic personality, she will feel fear and distress and she will respond differently. As someone is never $100 \%$ extravert or $100 \%$ neurotic, each personality factor will have a weight in determining how something is perceived and what decisions are being taken. Surely, Julies behaviour will not only be based

\footnotetext{
${ }^{1}$ Please read Egges et al. [11] and Kshirsagar et al. [12,13] for more details.
} 
on emotional concepts, but also on intellectual concepts. A dialogue system or intelligent agent will require concrete representations of concepts such as personality, mood and emotional state so that it can decide what behaviour it will portray $[4,14]$. As such, we need to define exactly what we mean by personality, mood, and emotion before we can simulate emotional perception, behaviour and expression.

\subsection{Basic Definitions}

An individual is an entity that is constantly changing. So, when we speak of an individual, we always refer to it relative to a time $t$. The moment that the individual starts existing is defined by $t=0$. The abstract entity that represents the individual at a time $t$ we will call $I_{t}$ from now on. An individual has a personality and an emotional state (we do not yet take mood into consideration). The model based on this assumption is called PE. The personality is constant and initialized with a set of values on $t=0$. The emotional state is dynamic and it is initialized to $\mathbf{0}$ at $t=0$. Thus we define $I_{t}$ as a tuple $\left(p, e_{t}\right)$, where $p$ represents the personality and $e_{t}$ represents the emotional state at time $t$. In our example, Julie will portray emotions (that change over time) based on what happens, but how she obtains these emotions and the behaviour that results from it, depends on a static part of her being, the personality.

There exist many personality models, each of them consisting of a set of dimensions, where every dimension is a specific property of the personality. Take for example the OCEAN model [3], which has five dimensions (Openness, Conscientiousness, Extraversion, Agreeableness, Neuroticism) or the PEN model [2] that has three dimensions. Generalizing from these theories, we assume that a personality has $n$ dimensions, where each dimension is represented by a value in the interval $[0,1]$. A value of 0 corresponds to an absence of the dimension in the personality; a value of 1 corresponds to a maximum presence of the dimension in the personality. The personality $p$ of an individual can then be represented by the following vector:

$$
p^{T}=\left[\alpha_{1} \ldots \alpha_{n}\right], \forall i \in[1, n]: \alpha_{i} \in[0,1]
$$

Emotional state has a similar structure as personality, but it changes over time. The emotional state is a set of emotions that have a certain intensity. For example, the OCC model defines 22 emotions, while Ekman [15] defines 6 emotions for facial expression classification. We define the emotional state $e_{t}$ as an $m$-dimensional vector, where all $m$ emotion intensities are represented by a value in the interval $[0,1]$. A value of 0 corresponds to an absence of the emotion; a value of 1 corresponds to a maximum intensity of the emotion. This vector is given as follows:

$$
e_{t}^{T}= \begin{cases}{\left[\beta_{1} \ldots \beta_{m}\right], \forall i \in[1, m]: \beta_{i} \in[0,1]} & \text { if } t>0 \\ \mathbf{0} & \text { if } t=0\end{cases}
$$


Furthermore, we define an emotional state history $\omega_{t}$ that contains all emotional states until $e_{t}$, thus:

$$
\omega_{t}=\left\langle e_{0}, e_{1}, \ldots, e_{t}\right\rangle
$$

An extended version of the PE model, the PME model, is given by including mood. We now define the individual $I_{t}$ as a triple $\left(p, m_{t}, e_{t}\right)$, where $m_{t}$ represents the mood at a time $t$. We define mood as a rather static state of being, that is less static than personality and less fluent than emotions [8]. Mood can be one-dimensional (being in a good or a bad mood) or perhaps multi-dimensional (feeling in love, being paranoid). Whether or not it is justified from a psychological perspective to have a multi-dimensional mood is not in the scope of this paper. However, to increase generality, we will provide for a possibility of having multiple mood dimensions. We define a mood dimension as a value in the interval $[-1,1]$. Supposing that there are $k$ mood dimensions, the mood can be described as follows:

$$
m_{t}^{T}= \begin{cases}{\left[\gamma_{1} \ldots \gamma_{k}\right], \forall i \in[1, k]: \gamma_{i} \in[-1,1]} & \text { if } t>0 \\ \mathbf{0} & \text { if } t=0\end{cases}
$$

Just like for the emotional state, there is also a history of mood, $\sigma_{t}$, that contains the moods $m_{0}$ until $m_{t}$ :

$$
\sigma_{t}=\left\langle m_{0}, m_{1}, \ldots, m_{t}\right\rangle
$$

\section{Emotional State and Mood Updating}

From perceptive input, an appraisal model such as OCC will obtain emotional information. This information is then used to update the mood and emotional state. We define the emotional information as a desired change in emotion intensity for each emotion, defined by a value in the interval $[0,1]$. The emotion information vector a (or emotion influence) contains the desired change of intensity for each of the $m$ emotions:

$$
a^{T}=\left[\delta_{1} \ldots \delta_{m}\right], \forall i \in[1, m]: \delta_{i} \in[0,1]
$$

The emotional state can then be updated using a function $\Psi_{e}\left(p, \omega_{t}, a\right)$. This function calculates, based on the personality $p$, the current emotional state history $\omega_{t}$ and the emotion influence $a$, the change of the emotional state. A second part of the emotion update is done by another function, $\Omega_{e}\left(p, \omega_{t}\right)$ that represents internal change (such as a decay of the emotional state). Given these two components, the new emotional state $e_{t+1}$ can be calculated as follows:

$$
e_{t+1}=e_{t}+\Psi_{e}\left(p, \omega_{t}, a\right)+\Omega_{e}\left(p, \omega_{t}\right)
$$


In the PME model (which includes the mood), the update process slightly changes. When an emotion influence has to be processed, the update now happens in two steps. The first step consists in updating the mood; the second step consists of updating the emotional state. The mood is updated by a function $\Psi_{m}\left(p, \omega_{t}, \sigma_{t}, a\right)$ that calculates the mood change, based on the personality, the emotional state history, the mood history and the emotion influence. The mood is internally updated using a function $\Omega_{m}\left(p, \omega_{t}, \sigma_{t}\right)$. Thus the new $\operatorname{mood} m_{t+1}$ can be calculated as follows:

$$
m_{t+1}=m_{t}+\Psi_{m}\left(p, \omega_{t}, \sigma_{t}, a\right)+\Omega_{m}\left(p, \omega_{t}, \sigma_{t}\right)
$$

The emotional state can then be updated by an extended function $\Psi_{e}^{\prime}$ that also takes into account the mood history and the new mood. The internal emotion update, which now also takes mood into account, is defined as $\Omega_{e}^{\prime}\left(p, \omega_{t}, \sigma_{t+1}\right)$. The new emotion update function is given as follows:

$$
e_{t+1}=e_{t}+\Psi_{e}^{\prime}\left(p, \omega_{t}, \sigma_{t+1}, a\right)+\Omega_{e}^{\prime}\left(p, \omega_{t}, \sigma_{t+1}\right)
$$

\section{Personality, Emotion and the OCC Model of Appraisal}

As OCC is the most widely accepted appraisal model, we will present some thoughts about how to integrate personality models with OCC. OCC uses goals, standards and attitudes. These three notions are for a large part domain dependent. As multi-dimensional personality models are domain-independent, we need to define the relationship between this kind of personality model and the personality model as it is used in OCC. We will choose an approach where we assume that the goals, standards and attitudes in the OCC model are fully defined depending on the domain. Our personality model will then serve as a selection criterion that indicates what and how many goals, structures and attitudes fit with the personality. Because the OCEAN model is widely accepted, we will use this model to illustrate our approach. For an overview of the relationship between OCEAN and the goals, standards, and attitudes, see Table 1. The intensity of each personality faction will determine the final effect on the goals, standards and attitudes.

\section{Application}

\subsection{Overview}

In order to demonstrate the model in a conversational context, we have built a conversational agent represented by a talking head. The update mechanisms of emotions and mood are implemented using a linear approach (using simple matrix representations and computations). The center of the application is a dialogue system, using Finite State Machines, that generates different responses 


\begin{tabular}{|l|l|l|l|}
\hline & Goals & Standards & Attitudes \\
\hline Openness & $\begin{array}{l}\text { making a shift of } \\
\text { standards in new sit- } \\
\text { uations }\end{array}$ & $\begin{array}{l}\text { attitude towards } \\
\text { new elements }\end{array}$ \\
\hline Conscientiousness & $\begin{array}{l}\text { abandoning and } \\
\text { adopting goals, } \\
\text { determination }\end{array}$ & & $\begin{array}{l}\text { willingness to com- } \\
\text { municate }\end{array}$ \\
\hline Extraversion & $\begin{array}{l}\text { abandoning and } \\
\text { adopting goals in in } \\
\text { favor of others }\end{array}$ & $\begin{array}{l}\text { compromising stan- } \\
\text { dards in favor of oth- } \\
\text { ers }\end{array}$ & \begin{tabular}{l} 
other people \\
\hline Neuroticism
\end{tabular} \\
\hline
\end{tabular}

Table 1. Relationship between OCEAN and OCC parameters.

based on the personality, mood and emotional state. The personality and emotion model is implemented using the 5 factors of the OCEAN model of personality, one mood dimension (a good-bad mood axis) and the 22 emotions from OCC plus 2 additional emotions (disgust and surprise) to have maximum flexibility of facial expression. A visual front-end uses the dialogue output to generate speech and facial expressions. The dialogue system annotates its outputs with emotional information. An example of such a response is (the tags consist of a joy emotion percentage of 58):

\section{| J058|Thanks! I like you too!}

\subsection{Visual Front-end}

Our visual front-end comprises of a 3D talking head capable of rendering speech and facial expressions in synchrony with synthetic speech. The facial animation system interprets the emotional tags in the responses, generates lip movements for the speech and blends the appropriate expressions for rendering in real-time with lip synchronization ${ }^{2}$. Facial dynamics are considered during the expression change, and appropriate temporal transition functions are selected for facial animation. We use MPEG-4 Facial Animation Parameters as low level facial deformation parameters [12]. However, for defining the visemes and expressions, we use the Principal Components (PCs) [13]. The PCs are derived from the statistical analysis of the facial motion data and reflect independent facial movements observed during fluent speech. The use of PCs facilitates realistic speech animation, especially blended with various facial expressions. We use available text-to-speech (TTS) software that provides phonemes with temporal information. The co-articulation rules are applied based on the algorithm of Cohen et al. [16]. The expressions are embedded in the text in terms of tags and associated intensities. An attack-sustain-decay-release type of envelope is applied for these

\footnotetext{
${ }^{2}$ For more details on expressive speech animation, see Kshirsagar et al. [13].
} 
expressions and they are blended with the previously calculated co-articulated phoneme trajectories. Periodic eye-blinks and minor head movements are applied to the face for increased believability. Periodic display of facial expression is also incorporated, that depends on the recent expression displayed with the speech, as well as the mood of the character.

\subsection{Example interaction}

As an example, we have developed a small interaction system that simulates Julie's behaviour. We have performed these simulations with different personalities, which gives different results in the interaction and the expressions that the face is showing. The interaction that takes place for an extravert personality (90\% extravert) is shown in Figure 2(b). An introvert personality (5\% extravert) significantly changes the interaction (see Figure 2(b)).

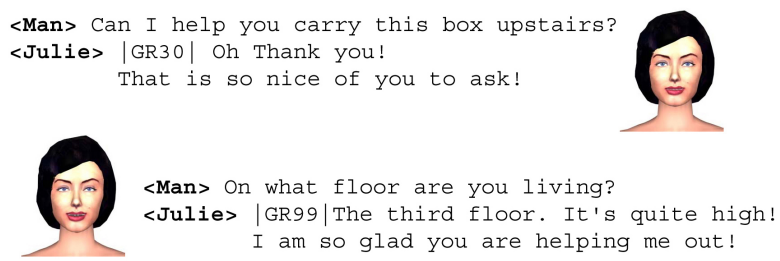

(a)

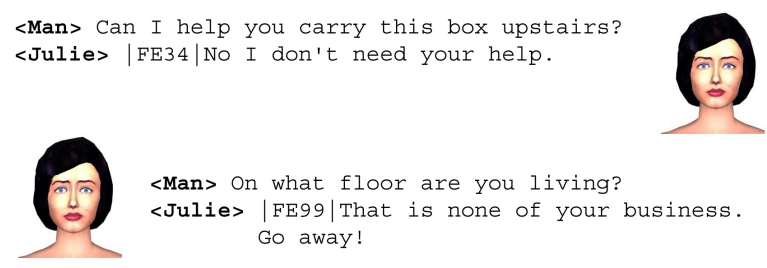

(b)

Fig. 2. Julie's behaviour as an extravert (a) and an introvert (b) personality.

\section{Conclusions and Future Work}

In this paper we have presented a basic framework for personality and emotion simulation. Subsequently we have shown how this framework can be integrated with an application such as an expressive MPEG-4 talking head with speech synthesis. Our future work will focus on user studies to validate the personality and emotion model that is used. We will also explore the possibility of having multiple mood dimensions. Furthermore we will explore how personality and emotion are linked to body behaviour and what computational methods are required to simulate this link. 


\section{Acknowledgment}

This research has been funded through the European Project MUHCI (HPRNCT-2000-00111) by the Swiss Federal Office for Education and Science (OFES).

\section{References}

1. Ortony, A., Clore, G.L., Collins, A.: The Cognitive Structure of Emotions. Cambridge University Press (1988)

2. Eysenck, H.J.: Biological dimensions of personality. In Pervin, L.A., ed.: Handbook of personality: Theory and research. New York: Guilford (1990) 244-276

3. Costa, P.T., McCrae, R.R.: Normal personality assessment in clinical practice: The NEO personality inventory. Psychological Assessment (1992) 5-13

4. Marsella, S., Gratch, J.: A step towards irrationality: Using emotion to change belief. In: Proceedings of the 1st International Joint Conference on Autonomous Agents and Multi-Agent Systems, Bologna, Italy (2002)

5. Johns, M., Silverman, B.G.: How emotions and personality effect the utility of alternative decisions: a terrorist target selection case study. In: Tenth Conference On Computer Generated Forces and Behavioral Representation. (2001)

6. André, E., Klesen, M., Gebhard, P., Allen, S., Rist, T.: Integrating models of personality and emotions into lifelike characters. In: Proceedings International Workshop on Affect in Interactions. Towards a New Generation of Interfaces. (1999)

7. Ball, G., Breese, J.: Emotion and personality in a conversational character. In: Proceedings of the Workshop on Embodied Conversational Characters. (1998) 8384 and $119-121$

8. Kshirsagar, S., Magnenat-Thalmann, N.: A multilayer personality model. In: Proceedings of 2nd International Symposium on Smart Graphics, ACM Press (2002) $107-115$

9. Velásquez, J.: Modeling emotions and other motivations in synthetic agents. In: Proceedings of AAAI-97, MIT Press (1997) 10-15

10. Moffat, D.: Personality parameters and programs. In: Lecture Notes in Artificial Intelligence : Creating Personalities for Synthetic Actors: Towards Autonomous Personality Agents. (1995)

11. Egges, A., Kshirsagar, S., Zhang, X., Magnenat-Thalmann, N.: Emotional communication with virtual humans. In: The 9th International Conference on Multimedia Modelling. (2003)

12. Kshirsagar, S., Garchery, S., Magnenat-Thalmann, N.: Feature Point Based Mesh Deformation Applied to MPEG-4 Facial Animation. In: Deformable Avatars. Kluwer Academic Publishers (2001) 33-43

13. Kshirsagar, S., Molet, T., Magnenat-Thalmann, N.: Principal components of expressive speech animation. In: Proceedings Computer Graphics International. (2001) 59-69

14. Elliott, C.D.: The affective reasoner: a process model of emotions in a multi-agent system. PhD thesis, Northwestern University, Evanston, Illinois (1992)

15. Ekman, P.: Emotion in the Human Face. Cambridge University Press, New York (1982)

16. Cohen, M.M., Massaro, D. In: Modelling co-articulation in synthetic visual speech. Springer-Verlag (1993) 139-156 